\title{
Propriétés physiques de la poudre de lait entier fabriquée selon la méthode de séchage à double effet
}

\author{
par \\ J. DE VILDER*
}

\section{INTRODUCTION}

La fabrication de poudre de lait a considérablement évolué ces dernières années sous l'influence de la hausse continue du coût de l'énergie et du fait de la demande de poudres de lait différant par leurs propriétés physiques et leurs caractéristiques fonctionnelles. Des modifications se sont imposées, autant en ce qui concerne la concentration du lait qu'en ce qui concerne l'atomisation du concentré.

Voici quelques années, l'emploi d'installations de concentration à triple ou à quadruple effet était généralisé dans l'industrie laitière. On les remplace aujourd'hui par des installations à quintuple, sextuple et même septuple effet, qui font nettement diminuer la consommation d'énergie.

La tendance à l'innovation s'est affirmée également dans le domaine du séchage par atomisation. A cause de la nécessité d'économiser de l'énergie et des exigences en matière de qualité, pratiquement chaque fabrique de poudre de lait se voit contrainte d'adopter le système de séchage dit à double effet, où un "lit fluidisé » est accouplé à une tour d'atomisation classique. Cette tour fonctionne alors avec une température de sortie moins élevée, donnant une poudre à plus haute teneur en eau. Le séchage final, jusqu'à la teneur en eau voulue, s'effectue ensuite dans le "lit fluidisé ". Jensen et Pisecky [6] ont signalé que ce procédé économisait de l'énergie. Selon Kragh [9] le séchage par atomisation à double effet procurerait une économie d'énergie et de frais d'investissement de 17 p. 100 par rapport au séchage classique.

La bibliographie ne fournit que peu de données sur l'influence exercée par le séchage à double effet sur les propriétés physiques

\footnotetext{
* Station Laitière de l'Etat, Melle. Centre de Recherches Agronomiques de l'Etat, Gand (Belgique).
} 
de la poudre de lait. King et Sanderson [8] ont mentionné dès 1970 que la solubilité de la poudre de lait s'améliore et que sa densité augmente quand la température de sortie de l'air sécheur de l'installation d'atomisation diminue. La teneur en eau de la poudre augmente cependant, ce qui nécessite un séchage final.

Mol et Jansen [10] ont rapporté que le séchage à double effet exerçait une influence positive sur la solubilité, le poids à l'emballage et le volume d'air restant. Selon Pisecky [12], le séchage à double effet procure un rendement énergétique plus favorable et une diminution des pertes de poudre. Il en résulte une amélioration de la qualité de la poudre, du fait de la basse teneur en air enclos, de la faible teneur en matière grasse libre et du poids à l'emballage élevé.

Dans ces publications, la comparaison porte toujours sur des poudres séchées respectivement par le procédé classique et par le procédé à double effet. Dans les recherches décrites ici, le séchage même, en "lit fluidisé ", et l'influence de ce séchage sur les propriétés physiques de poudres de lait entier ont été étudiés de plus près et l'attention s'est portée sur les caractéristiques suivantes : teneur en eau, indice de solubilité Admi, viscosité, teneur en matière grasse libre, poids à l'emballage, densité moyenne des particules, volume des vacuoles et porosité. Cette dernière caractéristique est fournie par la pénétration de l'azote dans la poudre et par le facteur de porosité $\gamma 10$.

\section{MATERIEL ET METHODES}

\section{II.1. Fabrication des poudres de lait}

Les poudres de lait entier fabriquées avaient une teneur en matière grasse de 26 à 26,8 p. 100 . La concentration s'effectuait par préchauffage à $85-90^{\circ} \mathrm{C}, 30 \mathrm{~s}$, jusqu'à une teneur en extrait sec de 46 à 50 p. 100. Une installation semi-industrielle d'atomisation par centrifugation, avec une capacité d'évaporation d'eau de 35 à $40 \mathrm{l} / \mathrm{h}$, était utilisée pour le séchage. La vitesse tangentielle de la turbine d'atomisation était de $135 \mathrm{~m} / \mathrm{s}$ dans tous les essais. La température de l'air d'entrée était toujours de 195 à $200^{\circ} \mathrm{C}$, mais la température de sortie variait d'un essai à l'autre, par modification du débit d'alimentation en concentré.

L'installation de séchage en "lit fluidisé ", accouplée à la tour de séchage pour le séchage à double effet, permettait d'opérer dans des conditions très variées. Le volume de la chambre de séchage et la température de l'air sécheur pouvaient être modifiés de façon à obtenir des temps de séchage de 3 à $8 \mathrm{~min}$ et des températures d'échauffement allant jusqu'à $105^{\circ} \mathrm{C}$. 
Dans chaque essai, la poudre de référence était toujours une poudre de lait fabriquée dans des conditions classiques avec des températures de l'air entrant de $195^{\circ} \mathrm{C}$ et de l'air sortant de $92^{\circ} \mathrm{C}$.

\section{II.2. Méthodes de détermination}

La teneur en eau a été déterminée par la méthode Karl Fisher (De Moor et al. [4]).

Pour déterminer la viscosité, on dissolvait $165 \mathrm{~g}$ de poudre dans $135 \mathrm{ml}$ d'eau à $23^{\circ} \mathrm{C}$. La dissolution s'effectuait dans un mélangeur Braun type MX32 fonctionnant pendant $20 \mathrm{~s}$ à la vitesse I et pendant $10 \mathrm{~s}$ à la vitesse III. La viscosité était déterminée à l'aide d'un viscosimètre Brookfield et de broches différentes selon la viscosité : broche 1 pour moins de $200 \mathrm{cPo}$, broche 2 pour 200 à $1000 \mathrm{cPo}$, broche 3 pour 1000 à $4000 \mathrm{cPo}$ et broche 4 pour toutes les viscosités dépassant $4000 \mathrm{cPo}$. La vitesse de rotation de la broche était toujours de 30 ou de 60 tours/min.

L'indice de solubilité Admi et la teneur en matière grasse libre étaient déterminés respectivement selon la méthode Admi [1] et la méthode Niro Atomizer [11]. La teneur en matière grasse libre est toujours exprimée en p. 100 de la teneur en matière grasse totale. Le poids d'emballage est calculé d'après le volume de $50 \mathrm{~g}$ de poudre après 600 coups de voluménomètre JEL ST2.

Les méthodes de Buma [3] étaient suivies pour les déterminations de la pénétration de l'azote, du facteur de porosité $(\gamma 10)$, de la densité moyenne des particules $(\mathrm{Dp})$ et du volume des vacuoles. La densité réelle, qu'il faut connaître pour déterminer le volume des vacuoles, était calculée en partant de la composition de la poudre de lait et des densités des différents composants du lait mentionnées par Buma [2].

Le degré d'homogénéisation de la matière grasse dans la poudre était mesuré au moyen de la pipette d'homogénéisation (Kiermeier et Lechner [7]) sur une solution à 13 p. 100 d'extrait sec.

\section{RESULTATS ET COMMENTAIRE}

\section{III.1. Poudres de lait classiques et poudres de lait obtenues sous séchage à double effet}

Avant de traiter de l'influence des différentes conditions de séchage à double effet, nous donnons un aperçu d'essais distincts, où six concentrés différents ont été séchés aussi bien par le procédé classique que par le procédé à double effet. Dans ce dernier procédé, la température de l'air sécheur à la sortie de la tour était de 74 à $78^{\circ} \mathrm{C}$ et les conditions de séchage en " lit fluidisé » étaient adaptées 
de façon que les poudres correspondantes aient des teneurs en eau équivalentes.

Le tableau 1 groupe les caractéristiques des différentes poudres. L'indice de solubilité Admi de la poudre séchée selon le procédé classique était relativement élevé dans deux essais, alors qu'il était très bas après le séchage à double effet. La viscosité des poudres redissoutes était aussi plus basse, dans tous les essais, après le séchage à double effet. Il en était de même de la teneur en matière grasse libre, tandis que le poids d'emballage et la densité moyenne des particules étaient plus élevés et que le volume des vacuoles était moins grand chez les poudres séchées selon le procédé à double effet. La pénétration de l'azote et le facteur de porosité $\gamma 10$ indiquent également que ces poudres sont moins poreuses. Ces résultats concordent étroitement avec les données des publications déjà citées et démontrent que le séchage à double effet améliore nettement la qualité des poudres.

\section{III.2. Séchage en " lit fluidisé ", considéré séparément}

Dans le séchage à double effet, la poudre a une teneur en eau relativement élevée (6 à 8 p. 100) quand elle arrive dans le " lit fluidisé " où elle subira le séchage final, jusqu'à la teneur en eau désirée. On peut toutefois se demander dans quelle mesure ce séchage final influe lui-même sur les propriétés de la poudre de lait.

Le tableau 2 reproduit les différentes caractéristiques de poudres prélevées en amont et en aval du " lit fluidisé ". Dans les différentes séries, les poudres ont été séchées en "lit fluidisé " jusqu'à une teneur en eau normale. La température de séchage appliquée était de 80 à $90^{\circ} \mathrm{C}$, pendant $4 \mathrm{~min}$, selon la teneur en eau initiale de la poudre venant de la tour.

L'échantillon $6 \mathrm{du}$ tableau 2 provient d'une fabrication où la température de sortie de l'air de séchage de l'installation d'atomisation était très basse (environ $65^{\circ} \mathrm{C}$ ). Du fait de la haute teneur en eau, il s'était produit une cristallisation du lactose entre le moment du prélèvement de l'échantillon et l'analyse. On a donc obtenu des valeurs inexactes pour la plupart des caractéristiques de l'échantillonnage avant le " lit fluidisé ". Ces chiffres ne figurent pas au tableau pour cette raison.

L'indice de solubilité des autres échantillons était initialement très bas et il n'a pas été influencé par les conditions de séchage en " lit fluidisé ". La viscosité après redissolution a toutefois toujours augmenté légèrement il n'est pas possible d'en déduire dans quelle mesure cette augmentation est due à la plus basse teneur en eau ou à des modifications des propriétés de la poudre, par exemple à une dénaturation de protéines.

Les chiffres montrent clairement que le séchage en « lit fluidisé » est, en soi, défavorable à la teneur en matière grasse libre. Ceci est 
TABLEAU 1

Poudres de lait entier, provenant d'un même concentré, mais séchées par le procédé classique ou par le procédé à double effet

\begin{tabular}{|c|c|c|c|c|c|c|c|c|c|c|}
\hline \multirow[b]{2}{*}{$\begin{array}{c}\text { Essai } \\
n^{\circ}\end{array}$} & \multirow[b]{2}{*}{$\begin{array}{c}\text { Conditions } \\
\text { de } \\
\text { séchage }\end{array}$} & \multicolumn{9}{|c|}{ Résultats } \\
\hline & & $\begin{array}{l}\text { Teneur } \\
\text { en eau }\end{array}$ & $\begin{array}{l}\text { Solubilité } \\
\text { indice } \\
\text { Admi }\end{array}$ & $\begin{array}{c}\text { Viscosité } \\
\text { cPo }\end{array}$ & $\begin{array}{l}\text { Teneur en } \\
\text { M.G.1.* } \\
\text { p. } 100 \\
\text { M.G tot.* }\end{array}$ & $\begin{array}{c}\text { Poids } \\
\text { d'emballage } \\
\mathrm{g} / \mathrm{cm}^{3}\end{array}$ & $\begin{array}{c}\text { Densité } \\
\text { moyenne } \\
\text { des } \\
\text { particules }\end{array}$ & $\begin{array}{c}\begin{array}{c}\text { Volume } \\
\text { des }\end{array} \\
\text { vacuoles } \\
\mathrm{cm}^{3} / 100 \mathrm{~g}\end{array}$ & $\begin{array}{l}\text { Pénétra- } \\
\text { tion } \mathrm{N}_{2} \\
\mathrm{~cm}^{3} / 100 \mathrm{~g}\end{array}$ & $\begin{array}{c}\text { Facteur } \\
\text { de } \\
\text { porosité } \\
\gamma 10\end{array}$ \\
\hline 1 & $\begin{array}{l}\text { classique } \\
\text { à double effet }\end{array}$ & $\begin{array}{l}2,50 \\
2,29\end{array}$ & $\begin{array}{l}0,55 \\
0,05\end{array}$ & $\begin{array}{r}1540 \\
730\end{array}$ & $\begin{array}{l}16,72 \\
12,42\end{array}$ & $\begin{array}{l}0,46 \\
0,55\end{array}$ & $\begin{array}{l}0,92 \\
1,08\end{array}$ & $\begin{array}{l}31,3 \\
15,6\end{array}$ & $\begin{array}{l}3,71 \\
3,17\end{array}$ & $\begin{array}{l}11,8 \\
20,3\end{array}$ \\
\hline 2 & $\begin{array}{l}\text { classique } \\
\text { à double effet }\end{array}$ & $\begin{array}{l}2,57 \\
2,49\end{array}$ & $\begin{array}{l}0,05 \\
0,05\end{array}$ & $\begin{array}{l}1510 \\
1200\end{array}$ & $\begin{array}{r}12,13 \\
8,48\end{array}$ & $\begin{array}{l}0,47 \\
0,56\end{array}$ & $\begin{array}{l}0,95 \\
1,09\end{array}$ & $\begin{array}{l}30,1 \\
19,8\end{array}$ & $\begin{array}{l}3,42 \\
0,70\end{array}$ & $\begin{array}{r}11,4 \\
3,5\end{array}$ \\
\hline 3 & $\begin{array}{l}\text { classique } \\
\text { à double effet }\end{array}$ & $\begin{array}{l}3,31 \\
2,92\end{array}$ & $\begin{array}{l}0,05 \\
0,05\end{array}$ & $\begin{array}{l}860 \\
685\end{array}$ & $\begin{array}{r}16,15 \\
9,94\end{array}$ & $\begin{array}{l}0,50 \\
0,56\end{array}$ & $\begin{array}{l}1,08 \\
1,11\end{array}$ & $\begin{array}{l}15,9 \\
12,1\end{array}$ & $\begin{array}{l}5,83 \\
2,70\end{array}$ & $\begin{array}{l}36,6 \\
22,3\end{array}$ \\
\hline 4 & $\begin{array}{l}\text { classique } \\
\text { à double effet }\end{array}$ & $\begin{array}{l}3,73 \\
3,43\end{array}$ & $\begin{array}{l}0,75 \\
0,05\end{array}$ & $\begin{array}{l}3200 \\
1515\end{array}$ & $\begin{array}{l}6,00 \\
4,09\end{array}$ & $\begin{array}{l}0,58 \\
0,64\end{array}$ & $\begin{array}{l}1,09 \\
1,12\end{array}$ & $\begin{array}{r}12,4 \\
9,7\end{array}$ & $\begin{array}{l}3,42 \\
0,70\end{array}$ & $\begin{array}{r}27,6 \\
7,8\end{array}$ \\
\hline 5 & $\begin{array}{l}\text { classique } \\
\text { à double effet }\end{array}$ & $\begin{array}{l}4,05 \\
3,80\end{array}$ & $\begin{array}{l}0,05 \\
0,05\end{array}$ & $\begin{array}{l}855 \\
620\end{array}$ & $\begin{array}{r}16,15 \\
9,94\end{array}$ & $\begin{array}{l}0,54 \\
0,60\end{array}$ & $\begin{array}{l}1,09 \\
1,11\end{array}$ & $\begin{array}{l}14,6 \\
13,2\end{array}$ & $\begin{array}{l}4,88 \\
1,31\end{array}$ & $\begin{array}{c}33,4 \\
9,91\end{array}$ \\
\hline 6 & $\begin{array}{l}\text { classique } \\
\text { à double effet }\end{array}$ & $\begin{array}{l}4,17 \\
3,71\end{array}$ & $\begin{array}{l}0,05 \\
0,05\end{array}$ & $\begin{array}{l}2330 \\
1560\end{array}$ & $\begin{array}{l}16,50 \\
10,00\end{array}$ & $\begin{array}{l}0,50 \\
0,58\end{array}$ & $\begin{array}{l}0,95 \\
1,08\end{array}$ & $\begin{array}{l}25,2 \\
15,6\end{array}$ & $\begin{array}{l}1,99 \\
0,78\end{array}$ & $\begin{array}{l}7,9 \\
5,0\end{array}$ \\
\hline
\end{tabular}

* Teneur en M.G.l. = teneur en matière grasse libre.

* Teneur en M.G. tot. = teneur en matière grasse tot. 
TABLEAU 2

Influence du séchage en "lit fluidisé » sur les propriétés de la poudre de lait entier

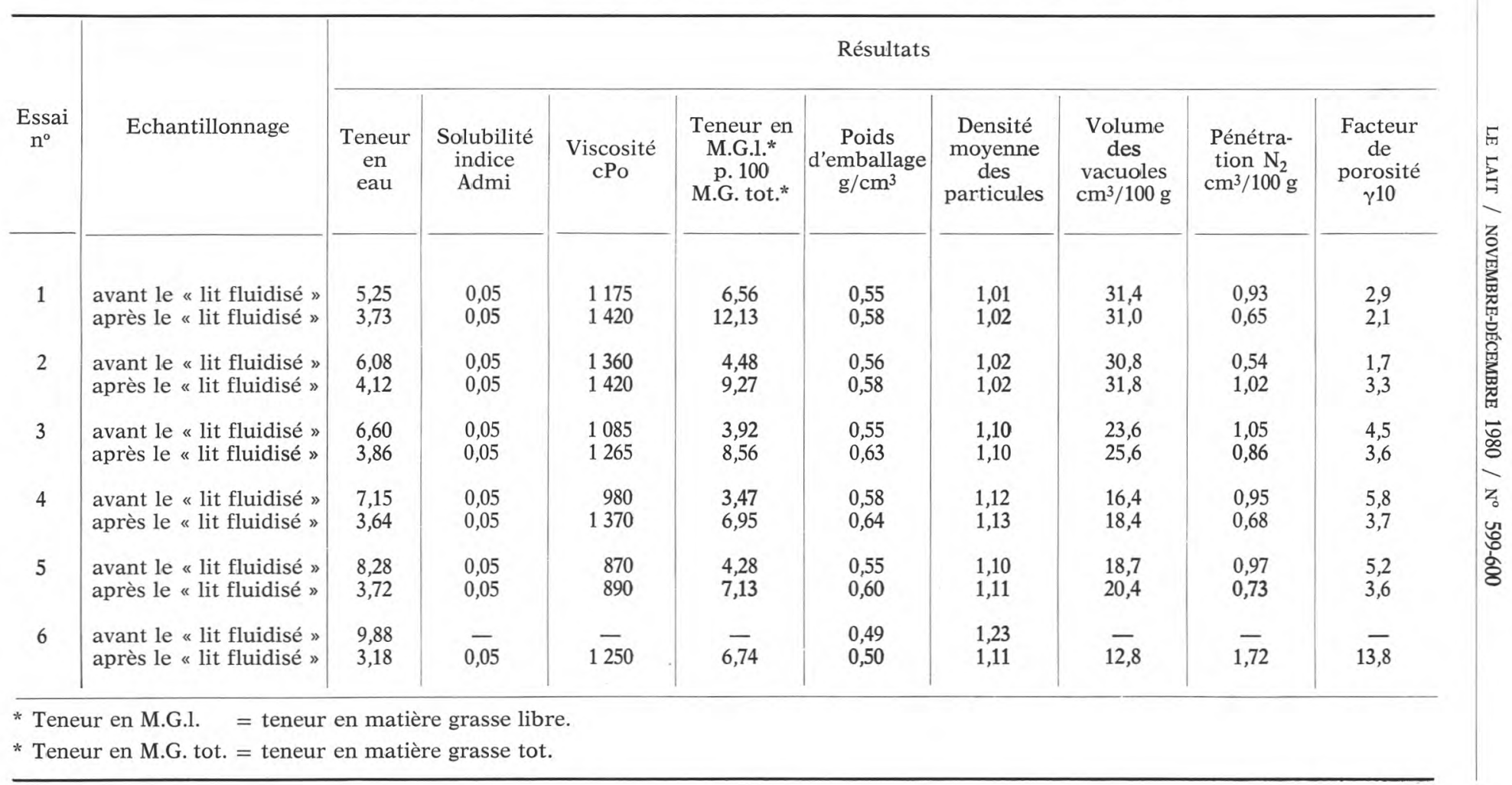


probablement imputable surtout à la désintégration, pendant le traitement en "lit fluidisé ", des agglomérats déjà formés. La désintégration de ces agglomérats est démontrée par l'augmentation de la densité d'emballage, du fait que les particules plus petites peuvent combler l'espace entre les plus gros grains. La densité moyenne des particules et le volume des vacuoles démontrent que la structure des particules de poudre individuelles n'est presque pas influencée par le séchage modéré en "lit fluidisé ».

La structure de la poudre avant le passage en " lit fluidisé " a déjà une grande influence sur la teneur en matière grasse libre et le volume des vacuoles du produit final. Les chiffres démontrent que la teneur en matière grasse libre et le volume des vacuoles du produit final diminuent dans la mesure où la teneur en eau de la poudre venant de la tour est plus élevée.

Les autres caractéristiques (pénétration de l'azote dans la poudre et facteur de porosité $\gamma 10$ ) n'ont pas fourni d'informations supplémentaires.

\section{III.3. Intensité du séchage en " lit fluidisé " pendant le séchage à double effet}

Les résultats ci-dessus ont toujours été obtenus au cours d'essais comportant un séchage final modéré en " lit fluidisé ". Il est néanmoins surtout apparu que la teneur en matière grasse libre en était influencée défavorablement. Du point de vue énergétique, il est toutefois plus avantageux d'utiliser le "lit fluidisé » pendant des temps de séchage plus courts, mais avec des températures de séchage plus élevées. Afin d'obtenir plus de données sur l'influence d'un séchage final plus intense, nous avons effectué trois séries d'essais à différentes intensités de séchage en « lit fluidisé ». Dans la première série d'essais, la poudre venant de la tour avait une teneur en eau moyenne de 6,15 p. 100 (tab. 3). Le séchage final durait $4 \mathrm{~min}$, à une température de 74 à $87^{\circ} \mathrm{C}$. Ce chauffage modéré a donné des poudres dont la teneur en eau variait entre 4,12 et 3,73 p.100. L'index de solubilité n'était pas influencé ; la teneur en matière grasse libre, la viscosité, le volume des vacuoles et la pénétration de l'azote dans les poudres augmentaient généralement quelque peu.

De plus hautes températures, 86 à $90^{\circ} \mathrm{C}$ pendant $4 \mathrm{~min}$, ont été appliquées en "lit fluidisé " au cours de la deuxième série d'essais. L'index de solubilité a augmenté ainsi légèrement à 95 et à $99^{\circ} \mathrm{C}$. La teneur en matière grasse libre et la viscosité ont augmenté dans tous les essais, le volume des vacuoles et la pénétration de l'azote dans trois essais sur quatre.

Dans la troisième série d'essais, des températures élevées ont été combinées avec un temps de séchage prolongé $(8 \mathrm{~min})$. Tous les 
TABLEAU 3

Influence de l'intensité du séchage en " lit fluidisé " sur les caractéristiques de la poudre de lait entier

\begin{tabular}{|c|c|c|c|c|c|c|c|c|c|c|c|c|c|}
\hline \multicolumn{2}{|c|}{$\begin{array}{l}\text { Séchage en } \\
\text { " lit fluidisé " }\end{array}$} & \multicolumn{2}{|c|}{$\begin{array}{l}\text { Teneur en eau } \\
\text { p. } 100\end{array}$} & \multicolumn{2}{|c|}{$\begin{array}{l}\text { Solubilité } \\
\text { indice } \\
\text { Admi }\end{array}$} & \multicolumn{2}{|c|}{$\begin{array}{c}\text { Teneur en M.G.l.* } \\
\text { p. } 100 \\
\text { M.G. tot.* }\end{array}$} & \multicolumn{2}{|c|}{ Viscosité cPo } & \multicolumn{2}{|c|}{$\begin{array}{l}\text { Volume des } \\
\text { vacuoles } \\
\mathrm{cm}^{3} / 100 \mathrm{~g}\end{array}$} & \multicolumn{2}{|c|}{$\begin{array}{l}\text { Pénétration } \mathrm{N}_{2} \\
\mathrm{~cm}^{3} / 100 \mathrm{~g}\end{array}$} \\
\hline durée & ${ }^{\circ} \mathrm{C}$ & avant & après & avant & après & avant & après & avant & après & avant & après & avant & après \\
\hline $4 \mathrm{~min}$ & $\begin{array}{l}74 \\
81 \\
85 \\
87\end{array}$ & 6,15 & $\begin{array}{l}4,12 \\
3,91 \\
3,73 \\
3,73\end{array}$ & 0,05 & $\begin{array}{l}0,05 \\
0,05 \\
0,05 \\
0,05\end{array}$ & 4,27 & $\begin{array}{l}6,24 \\
5,98 \\
7,23 \\
7,77\end{array}$ & 1135 & $\begin{array}{l}1710 \\
1565 \\
1485 \\
1420\end{array}$ & 30,5 & $\begin{array}{l}30,8 \\
31,0 \\
31,7 \\
31,7\end{array}$ & 0,66 & $\begin{array}{l}1,02 \\
0,78 \\
1,00 \\
0,65\end{array}$ \\
\hline $4 \min$ & $\begin{array}{l}86 \\
91 \\
95 \\
99\end{array}$ & 5,28 & $\begin{array}{l}2,14 \\
2,03 \\
1,99 \\
1,59\end{array}$ & 0,05 & $\begin{array}{l}0,05 \\
0,05 \\
0,10 \\
0,20\end{array}$ & 8,09 & $\begin{array}{l}15,53 \\
14,94 \\
21,22 \\
18,39\end{array}$ & 980 & $\begin{array}{l}2300 \\
2200 \\
2450 \\
3300\end{array}$ & 16,8 & $\begin{array}{l}15,6 \\
17,8 \\
17,7 \\
25,8\end{array}$ & 3,38 & $\begin{array}{l}3,15 \\
6,33 \\
6,05 \\
6,70\end{array}$ \\
\hline $8 \mathrm{~min}$ & $\begin{array}{l}91 \\
96 \\
96 \\
98\end{array}$ & 6,19 & $\begin{array}{l}2,04 \\
2,06 \\
1,97 \\
2,20\end{array}$ & 0,10 & $\begin{array}{l}0,20 \\
0,10 \\
0,50 \\
0,30\end{array}$ & 3,49 & $\begin{array}{l}16,40 \\
14,71 \\
15,16 \\
12,56\end{array}$ & 1280 & $\begin{array}{l}1930 \\
1600 \\
5100 \\
3700\end{array}$ & 13,9 & $\begin{array}{l}21,4 \\
18,4 \\
21,7 \\
19,6\end{array}$ & 0,06 & $\begin{array}{l}2,69 \\
2,13 \\
2,22 \\
1,25\end{array}$ \\
\hline $\begin{array}{l}\text { Avant }= \\
\text { Après }=\end{array}$ & $\begin{array}{l}\text { intil } \\
\text { intill }\end{array}$ & $\begin{array}{l}\text { rélevé } \\
\text { rélevé }\end{array}$ & $\begin{array}{l}\text { mont } \\
\text { val d }\end{array}$ & $\begin{array}{l}\text { «lit } \\
\text { lit flu }\end{array}$ & é ». & & $\begin{array}{l}\text { Teneu } \\
\text { Teneu }\end{array}$ & $\begin{array}{l}\text { M.G. } \\
\text { M.G. }\end{array}$ & $\begin{array}{l}=\text { ten } \\
=\text { ten }\end{array}$ & $\begin{array}{l}\text { en } m \\
\text { en } m\end{array}$ & $\begin{array}{l}\text { e grass } \\
\text { e gras: }\end{array}$ & $\begin{array}{l}\text { ibre. } \\
\text { ot. }\end{array}$ & \\
\hline
\end{tabular}


paramètres étudiés en ont été modifiés nettement dans un sens négatif.

On peut déduire de ces résultats que l'influence favorable d'une diminution de la température de sortie est plus ou moins maintenue par un séchage final modéré en "lit fluidisé ". Un séchage final intense, par contre, influe sur la structure physique de la poudre, ce qui a un effet défavorable sur sa teneur en matière grasse libre et sur sa viscosité après redissolution. Ces essais indiquent qu'un séchage final à $95^{\circ} \mathrm{C}$ et d'une durée maximale de 4 min était acceptable.

\section{III.4. Séchage à double effet de poudres fabriquées à partir de concentrés homogénéisés}

Une des principales améliorations résultant du séchage à double effet est incontestablement l'abaissement de la teneur en matière grasse libre. On sait que l'homogénéisation du concentré fait également diminuer cette teneur (De Vilder et Martens [5]). Le tableau 4 reproduit les résultats de deux fabrications partant de concentrés à respectivement 46 et 50,1 p. 100 d'extrait sec. La pression d'homogénéisation était de 120 et de 150 bars* $^{*}$ et la température du concentré juste avant l'atomisation de $65^{\circ} \mathrm{C}$.

Il ressort de la première série d'essais que l'influence de l'homogénéisation sur la teneur en matière grasse libre est plus grande en cas de séchage classique que l'influence de séchage en "lit fluidisé » d'un concentré non homogénéisé. La valeur la plus basse est toutefois obtenue quand on combine l'homogénéisation du concentré et le séchage en "lit fluidisé ». L'indice de solubilité est aussi très bas dans ce cas.

Dans la deuxième série de fabrication, le concentré avait une teneur en extrait sec plus élevée $(50,1 \mathrm{p} .100)$. La teneur en matière grasse libre de la poudre fabriquée selon le procédé classique, sans homogénéisation, était de ce fait très basse. L'homogénéisation du concentré a encore accentué la diminution de la teneur en matière grasse libre, mais a influé défavorablement sur l'indice de solubilité. L'application conjointe de l'homogénéisation et du séchage en "lit fluidisé " a exercé une influence positive minime sur la teneur en matière grasse libre. L'indice de solubilité Admi restait toutefois bas. Les essais n'ont pas permis de faire une distinction entre les poudre fabriquées sous des pressions d'homogénéisation respectives de 120 et 150 bars.

L'influence de la pression d'homogénéisation sur la viscosité de la poudre redissoute s'est révélée plutôt minime. Dans la première série, la viscosité de la poudre de fabrication classique (non homo-

* 10 bars = $1 \mathrm{MPa}$. 
Influence de l'homogénéisation sur les propriétés de poudres de lait séchées par le procédé classique ou séchées par le procédé à double effet

\begin{tabular}{|c|c|c|c|c|c|c|c|c|}
\hline \multirow[b]{2}{*}{ Fabrication } & \multicolumn{4}{|c|}{ Série 1 : concentré à 46 p. 100} & \multicolumn{4}{|c|}{ Série 2 : concentré à 50,1 p. 100} \\
\hline & $\begin{array}{l}\text { Teneur } \\
\text { en eau }\end{array}$ & $\begin{array}{l}\text { Solubilité } \\
\text { indice } \\
\text { Admi }\end{array}$ & $\begin{array}{c}\text { Viscosité } \\
\text { cPo }\end{array}$ & $\begin{array}{c}\text { Teneur en } \\
\text { M.G.l.* } \\
\text { p } 100 \\
\text { M.G. tot.* }\end{array}$ & $\begin{array}{l}\text { Teneur } \\
\text { en eau }\end{array}$ & $\begin{array}{l}\text { Solubilité } \\
\text { indice } \\
\text { Admi }\end{array}$ & $\begin{array}{c}\text { Viscosité } \\
\text { cPo }\end{array}$ & $\begin{array}{l}\text { Teneur en } \\
\text { M.G.1* } \\
\text { p. } 100 \\
\text { M.G. tot.* }\end{array}$ \\
\hline Classique N.H.* & 2,54 & 0,55 & 1540 & 16,72 & 3,54 & 0,05 & 810 & 8,44 \\
\hline Classique H 150 bars** & 2,63 & 0,60 & 920 & 8,32 & 3,47 & 0,30 & 910 & 5,17 \\
\hline " Lit fluidisé » N.H. & 2,29 & 0,05 & 730 & 12,42 & 3,18 & 0,05 & 790 & 6,33 \\
\hline " Lit fluidisé » H 120 bars** & 2,04 & 0,05 & 1010 & 5,02 & 3,33 & 0,05 & 830 & 5,12 \\
\hline « Lit fluidisé » H 150 bars & 2,08 & 0,05 & 990 & 6,44 & 3,39 & 0,05 & 800 & 4,88 \\
\hline
\end{tabular}

* N.H. = concentré non homogénéisé.

** H 150 bars = concentré homogénéisé en simple effet sous une pression de 150 bars.

* H 120 bars = concentré homogénéisé en simple effet sous une pression de 120 bars.

* Teneur en M.G.l. = teneur en matière grasse libre.

* Teneur en M.G. tot. = teneur en matière grasse tot. 
généisée) était plus grande que celle de la poudre fabriquée avec séchage à double effet (également non homogénéisée).

Il découle de ces essais que l'homogénéisation du concentré abaisse la teneur en matière grasse libre même en cas de séchage à double effet. Son influence est d'autant plus nette que cette caractéristique est plus élevée dans la poudre de fabrication classique. On peut admettre d'une façon générale que l'effet est minime si les concentrés traités ont des teneurs en extrait sec supérieures à 50 p. 100. Dans ces cas, le séchage à double effet peut abaisser nettement l'indice de solubilité Admi. Il est donc recommandé de combiner l'homogénéisation avec le séchage à double effet si la poudre doit satisfaire à des exigences particulièrement sévères, par exemple moins de 5 p. 100 de matière grasse libre et un indice de solubilité Admi inférieur à $0,10 \mathrm{ml}$.

\section{R é $\mathbf{s}$ u m é}

Dans des conditions de travail normales, le séchage à double effet exerce une influence favorable sur les propriétés physiques et sur la structure de la poudre de lait entier. Le faible indice de solubilité Admi et la très basse teneur en matière grasse libre sont dans ce cas particulièrement intéressants.

Le séchage en "lit fluidisé » est cependant défavorable, en soi, à la teneur en matière grasse libre. Dans le séchage à double effet normal, cette influence défavorable est largement compensée par la très basse teneur en matière grasse libre de la poudre avant son passage en " lit fluidisé ». En cas de séchage final intense, elle sera cependant nuisible à la structure physique des poudres, en faisant augmenter le volume des vacuoles, la viscosité et, surtout, la teneur en matière grasse libre et l'indice de solubilité. Un séchage final de 4 min à $95^{\circ} \mathrm{C}$ a semblé être le maximum acceptable au cours des essais décrits ici.

L'homogénéisation du concentré afin d'abaisser la teneur en matière grasse libre peut encore être utile quand on recourt au séchage à double effet. Ce mode de séchage annule en outre l'influence négative de l'homogénéisation sur l'indice de solubilité Admi.

On peut conclure d'une façon générale que, dans la fabrication de poudre de lait entier à humidité normale (3 à 4 p. 100), le séchage à double effet exerce une influence positive sur les propriétés physiques de cette poudre.

Les résultats ont été obtenus dans des essais à l'échelle pilote. On retrouvera probablement les mêmes tendances dans des conditions de fabrication industrielles. Les valeurs absolues mentionnées pouvant différer d'une installation à l'autre. 


\section{Remerciements}

L'auteur remercie M. H. De Ridder, ingénieur industriel, membre du Groupe de Travail pour l'amélioration des produits laitiers, de sa précieuse collaboration.

\section{S u m m a r y}

THE PHYSICAL PROPERTIES OF TWO-STAGE DRIED WHOLE MILK POWDER

Under normal working conditions two-stage drying favourably affects the physical characteristics and the structure of whole milk powder. Very important are the low Admi solubility index and the very slight free fat content obtained.

Fluid bed drying itself, however, unfavourably affects the free fat content. On normal two-stage drying this unfavourable influence is largely compensated by the very low free fat content of the powder before its fluid bed drying. On very intensive after drying this will unfavourably affect the physical structure of the powders, which results in an increase not only in vacuole volume and viscosity, but also in free fat content and solubility index. Experience of our own has shown that an after drying for $4 \mathrm{~min}$ at $95^{\circ} \mathrm{C}$ is the permissible maximum.

On two-stage drying, a decrease in free fat content can still be obtained by homogenization of the concentrate. In addition, this drying technique neutralizes the negative effect produced by the homogenization on the Admi solubility index.

It can be generally concluded that a two-stage drying favourably affects the physical characteristics of whole milk powder which have a normal moisture content of between 3 and 4 p. 100 .

The results were recorded during research on a pilot scale. Under industrial conditions the same trends are likely to be found. The absolute values mentioned in this paper may, however, differ from plant to plant.

Reçu pour publication en juin 1980.

\section{Bibliographie}

[1] Admi (1971). - Determination of Solubility Index. Standards for Grades of Dry Milks. American Dry Milk Institute Bulletin, 916, 2, 26.

[2] Buma (T. J.) (1965). - The true density of spray milk powder and of certain constituents. Neth. Milk Dairy J., 19, 249.

[3] Buma (T. J.) (1971). - Free fat in spray-dried whole milk. The relation between free-fat content and particle porosity of spray-dried whole milk. Neth. Milk Dairy J., 25, 123. 
[4] De Moor (H.), De Cordier (J.) en Hendrickx (H.) (1967). - Bepaling van het vochtgehalte van volle verstuivingspoeder met de Karl-Fischer-titratie metode. Meded. Rijksfac. Landbouwwet. Gent, 32, 1, 49.

[5] De Vilder (J.) and Martens (R.) (1974). - Influence of the homogenization of concentrated milk on some wholemilk powder characteristics. $X I X$. Int. Dairy Congr., 1 E., 609.

[6] Jensen (D. J.) und Pisecky (J.) (1975). - Effektive Energieausnutzung bei der Herstellung von Milkpulver. Die Molkerei-Zeitung, Welt der Milch, $29,14,362$.

[7] Kiermeier (F.) und Lechner (E.) (1973). - Bestimmung des Homogenisierungseffektes. Milch und Milcherzeugnisse, Verlag Paul Parey, Berlin 314.

[8] King (D. W.) et SANDERson (W. B.) (1970). - Variables de fabrication agissant sur l'indice de solubilité et sur la densité de la poudre de lait écrémé " spray ". XVIII Congr. Intern. Lait., $1 \mathrm{~F}, 273$.

[9] KRAGH (O. T.) (1977). - A new generation of milk drying plants. An analysis of the two-stage drying process. Nordeuropaeisk mejeri-tidsskrift, 1-46.

[10] Mol (J. J.) en JANSEN (L. A.) (1974). - De oplosbaarheid van vol melkpoeder. Officieel orgaan FNZ, 66, 8, 190.

[11] Niro Atomizer (1978). - Determination of Free Fat on the Surface of Milk Powder Particles. Analytical Methods for Dry Milk Products, 4, 47.

[12] Pisecky (J.) (1974). - Milk droplets: their creation and drying. The World Galaxy, 5, 27. 